\title{
La enseñanza del portugués en noroeste de México desde una perspectiva glotopolítica
}

\author{
Teaching Portuguese in Northwest Mexico from a glotopolitical perspective \\ O ensino de português no noroeste do México a partir de uma perspectiva glotopolítica
}

Recibido: 20/12/2021 | Revisado: 27/12/2021 | Acepto: 31/12/2021 | Publicado: 08/01/2022

\author{
David Guadalupe Toledo-Sarracino \\ ORCID: https://orcid.org/0000-0001-8795-2909 \\ Universidad Autónoma de Baja California, México \\ E-mail: dtoledo@uabc.edu.mx \\ Gabriel Leopoldino dos Santos \\ ORCID: https://orcid.org/0000-0002-3030-1969 \\ Instituto Federal de São Paulo, Brasil \\ E-mail: gsantos18@gmail.com
}

\begin{abstract}
Resumen
El siguiente trabajo describe la historia de la enseñanza del portugués en Baja California (México) y la implementación de una política lingüística explícita en la Universidad Autónoma de Baja California como una estrategia de internacionalización de una universidad líder en el noroeste de México. UABC participa en los programas de becas de intercambio estudiantil tales como Eramus, grupo CAMBRIA y Grupo Santander y es hasta el 2010 cuando se diseña el programa de portugués lengua extranjera (PLE) como fomento al multilingüismo hacia la internacionalización de sus programas educativos en el contexto internacional incluyendo a Brasil y Portugal. Este estudio de tipo descriptivo, analiza las narrativas de expertos del portugués involucrados los inicios de la enseñanza del PLE en la región fronteriza desde un enfoque glotopolítico de Guespin, Marcellesi y Lagares. Con ello, se busca documentar al lenguaje desde un elemento esencial de la sociocultura y la interacción entre sus hablantes. Los resultados indican, por un lado, el estado actual de la enseñanza de esta lengua extranjera y el futuro que visualizan dichos actores en los procesos de internacionalización y enseñanza de lenguas extranjeras en la región fronteriza (México-Estados Unidos). Por el otro, los resultados indican un proceso eminentemente político, el cual ha producido impactos en las relaciones entre las lenguas en el espacio de funcionamiento de lenguas considerado.
\end{abstract}

Palabras clave: Glotopolítica; Portugués como lengua extranjera; Internacionalización; Políticas lingüísticas.

\begin{abstract}
This paper describes and analyzes the history of Portuguese teaching in Baja California (Mexico), as well as the implementation of an explicit language policy at the Universidad Autónoma de Baja California (UABC) as part of an internationalization strategy conducted by a leading university in northwestern Mexico. UABC participates in students' exchange scholarship programs such as Erasmus, CAMBRIA Group and Santander Group. It was only in 2010 that the Portuguese as a foreign language program was designed to encourage multilingualism and the internationalization of its educational programs in the international context, including Brazil and Portugal. This descriptive-analytical study analyzes, from a glotopolitical focus from Guespin, Marcellesi and Lagares. the narratives of Portuguese experts involved in the beginnings of Portuguese in the border region. With this, we seek to understand language as an essential element for society/culture and for the interaction between its speakers. The results indicate, on the one hand, the current state of teaching this foreign language and the future of the actors involved in the processes of internationalization and teaching of foreign languages in the border region (Mexico-United States). On the other hand, the results point to an eminently political process, which has produced impacts on the relations among languages in the considered space of functioning of languages.
\end{abstract}

Keywords: Glotopolitics; Portuguese as a foreign language; Internationalization; Language policy.

\section{Resumo}

O presente trabalho descreve e analisa a história do ensino de português em Baja California (México), bem como a implementação de uma política linguística explícita na Universidad Autónoma de Baja California (UABC) como uma estratégia de internacionalização de uma universidade líder no noroeste do México. A UABC participa de programas de concessão de bolsas de intercâmbio estudantil, tais como Erasmus, grupo CAMBRIA e Grupo Santander. É somente em 2010 que é desenhado o programa de português língua estrangeira (PLE) como fomento ao multilinguismo e à internacionalização de seus programas educativos no contexto internacional, incluindo o Brasil e Portugal. Esse estudo de tipo descritivo-analítico, analisa, a partir de um enfoque glotopolítico Guespin, Marcellesi e Lagares. As narrativas de 
especialistas em português envolvidos nos inícios do ensino do PLE na região fronteiriça. Com isso, busca-se compreender a linguagem como um elemento essencial para a sociedade/cultura e para a interação entre seus falantes. Os resultados indicam, por um lado, o estado atual do ensino dessa língua estrangeira e o futuro que os atores envolvidos nos processos de internacionalização e ensino de línguas estrangeiras na região de fronteira (México-Estados Unidos). Por outro lado, os resultados apontam para um processo eminentemente político, o qual produziu impactos nas relações entre as línguas no espaço de funcionamento de línguas considerado.

Palavras-chave: Glopolítica; Portugués como língua estrangeira; Internacionalização; Política linguística.

\section{Introducción}

El presente estudio objetiva realizar un estudio descriptivo-analítico acerca de los procesos de implementación de la enseñanza del portugués en Baja California en México, en sí, se enuncia una política explícita de internacionalización de la Universidad Autónoma de Baja California (UABC). Consideraremos que dicha política en materia de enseñanza del portugués como lengua extranjeras (PLE) representa la materialización de una acción glotopolítica, una vez que decisiones concernientes a la política lingüística de una institución, como la que acabamos de nombrar, pasa por atravesamientos discursivos acerca de lo qué es una lengua, de lo qué es el multi/plurilingüismo, de lo significa hablar una lengua extranjera no solo desde el punto de vista cognitivo, sino también político-social, etc.

La discusión que se presenta en este texto se justifica porque se buscará mostrar que toda decisión que afecte el lenguaje es una decisión eminentemente política, y no, como se podría pensar, desinteresada. De este modo, al trazar un recorrido que ilustre los procesos de implementación de la enseñanza del portugués en la frontera norte México-Estados, pondremos en escena contornos específicos de tomas de decisión que alteran la situación lingüística de ese espacio a la medida que, con la inserción de la lengua portuguesa en el catálogo de las lenguas enseñables, de alguna manera se alteran las relaciones entre las lenguas — sus juegos de fuerzas - que son habladas/estudiadas/enseñadas en este espacio fronterizo..

De modo a comprender en qué sentido la enseñanza de una lengua extranjera por una institución universitaria es una acción glotopolítica, en el siguiente apartado se describe a la glotopolítica como un campo específico de conocimiento sobre las lenguas/el lenguaje.

\section{Entre lo lingüístico y lo político: La Glotopolítica como campo del conocimiento sobre el lenguaje}

La glotopolítica surge en el marco intelectual francés en el último cuarto del siglo XX con la propuesta de seguir adelante con las propuestas sociolingüísticas de mediados de ese mismo siglo en las que se propone pensar las relaciones entre el lenguaje, la política y la sociedad. En realidad, las cuestiones entre la lengua y la política nacen como una ramificación de las cuestiones que se ponían, desde hacía algún tiempo, entre la lengua y la sociedad.

Es, así, en ese contexto de las ideas lingüísticas que Jean B. Marcellesi y Louis Guespin definen el campo de estudio de la Glotopolítica como constituído por:

[...] las diversas formas en que una sociedad actúa sobre el lenguaje, sea o no consciente de ello: tanto sobre la lengua, cuando por ejemplo una sociedad legisla respecto de los estatutos recíprocos de la lengua oficial y las lenguas minoritarias; como sobre el habla, cuando reprime tal o cual uso en uno u otro; o sobre el discurso cuando la escuela decide convertir en objeto de evaluación la producción de un determinado tipo de texto (Marcellesi; Guespin, 1986).

De lo que se acaba de exponer, destacamos que es interés de ese campo de estudios sobre el lenguaje las "diversas formas en que una sociedad actúa sobre el lenguaje". Hay, entonces, una dimensión práctica —en el sentido de una praxis— que interfiere 
en el modo como una lengua funciona en determinado espacio. Entre las formas de actuación social sobre el lenguaje, podemos mencionar desde la publicación de gramáticas y diccionarios hasta la publicación de manuales didácticos, programas de enseñanza de lenguas y proyectos de implementación de una política lingüística institucional.

La autoridad más importante de esa perspectiva teórica en Latinoamérica es la investigadora argentina Elvira Narvaja de Arnoux, de la Universidad de Buenos Aires. Según Arnoux (2008, p. 12):

El interés de la perspectiva que plantean [Marcellesi y Guespin (1986)] es, por un lado, que se restringe al estudio de las acciones sobre la lengua o la relación entre lenguas, sino que se abre a las múltiples intervenciones que se pueden ejercer en el espacio del lenguaje y que inciden o se exponen, asimismo, en los comportamientos. Por otro lado, anuncia la diversidad de lugares de discurso en los que se pueden indagar las políticas lingüísticas [...].

La autora menciona como objetos de interés glotopolítico lo que denomina "intervenciones glotopolíticas en sentido estricto", es decir, legislaciones, resoluciones, informes, etc., sino también gramáticas, diccionarios, textos escolares, manuales de estilo, discursos político-pedagógicos, memorias, biografías, ensayos periodísticos, grabaciones, películas, entre otras publicaciones. Es así que proponemos pensar la historia de implementación del portugués como lengua extranjera en Baja California (caso específico para la UABC) como un hecho glotopolítico.

\section{Antecedentes de una política lingüística de la lengua portuguesa en México}

Almeida Filho (1992) y Dalpiaz y Stanzani (2012) describen que el portugués como lengua extranjera (PLE) tiene una historia de cinco siglos en América que se extiende, desde los primeros esfuerzos religiosos jesuitas, por la enseñanza de la lengua a miembros de nacionales indígenas en Brasil con propósitos de evangelización. Es hasta la década de los sesenta que llega a México como una actividad sociocultural para construir una comunidad universitaria que aprende lenguas extranjeras. Sin duda en los sesenta y setenta se extendió a lo largo de toda Latinoamérica con un mayor crecimiento de estudiantes universitarios la manera cómo enseñar la lengua, el diseño de materiales y los cursos de formación de profesores a lo largo de toda América Latina.

Colin Rodea (2021) señala que, con la llegada de brasileños a México, a consecuencia del golpe de estado de 1964 en Brasil, México se convierte en un país de exilio para las comunidades sudamericanas y, como resultado, hubo un grupo de profesores nativo hablantes de la lengua portuguesa que enriquecieron el mosaico lingüístico en la Ciudad de México con la enseñanza del PLE en la UNAM. En 1975 se inaugura el Centro Cultural Brasil-México (CCBM) en la Ciudad de México perteneciente al grupo de la Red Brasil Cultural, espacio cultural para la difusión de la lengua y la cultura con apoyo de la Embajada de Brasil en México. En 1967, se inician los trabajos de enseñanza de la lengua portuguesa en la UNAM por las profesoras Pia Mehner y después Valquiria Wey quienes fueron las pioneras de la enseñanza de la lengua portuguesa en México.

En el año que inicia el programa de PLE en el entonces Centro de Enseñanza de Lenguas Extranjeras (CELE), ubicado en la Facultad de Filosofía y Letras, se comenzó a enseñar la lengua con el espíritu de diversidad y diálogo en la comunidad estudiantil de la Universidad Nacional Autónoma de México (UNAM). En la actualidad es en la Escuela Nacional de Lenguas, Lingüística y Traducción (ENALLT) que se enseña esta lengua como lengua adicional y en el marco del programa de formación de profesores (Herrera, 2006 y UNAM, 2021). Más adelante para 1977 se comenzó a enseñar PLE en la Escuela Nacional de Estudios Profesional (ENEP) Aragón y en 1980 en la ENEP Acatlán en 1980 (lo que hoy es la Facultad de Estudios Superiores (FES) en la UNAM.

La UNAM se benefició al contar con una planta de profesores pioneros que fueron el asidero académico de la enseñanza de la lengua lusófona en México, tales como los profesores Helena da Silva, Francisco Lage Pessoa y Bruno Alves de Castro, quienes 
en 1982 diseñaron el Curso Activo de portugués. Ese programa evolucionó, siendo el cimiento para fortalecer la formación de profesores en el Departamento de Lingüística Aplicada del entonces Centro de Enseñanza de Lenguas Extranjeras (hoy ENALLT) y de enseñanza del portugués en México.

En la década de los noventa, se originó el boom del tratado comercial del cono sur denominado Mercosur (Mercado Común del Sur). Ese proyecto económico, político, social y cultural representó el posicionamiento en un bloque sudamericano de 4 países que se unificarán sus economías para el bien común del continente, como el polo económico-sudamericano de vanguardia que sería el contrapeso del Tratado de Libre Comercio de América del Norte (TLCAN). Sin duda, desde México ya se realizaban los esfuerzos académicos por posicionar la enseñanza del portugués vislumbrando los inicios de las bases sólidas de la enseñanza de esta lengua en el contexto universitario mexicano.

El 26 de marzo de 1991 se firmó el "Tratado de Asunción”, lo que sería el parteaguas de la alianza política de Argentina, Brasil, Paraguay y Uruguay, países emergentes con fuerza política que de alguna manera iniciaría sería el principio del neoliberalismo de los mercados en el mundo e interrelacionar los alcances de la globalización. Es hasta 1994 que comienza su funcionalidad y los bloques de educación, salud, cultura y residencia.

A partir de la integración del Mercosur, se comenzó a preparar al universitario para competir en los contextos educativos sudamericanos y se asumió que el portugués tomaría fuerza en todo Latinoamérica desde el Cono Sur hasta Canadá. Las universidades públicas y privadas diseñaron estrategias de incorporar programas de aprendizaje de lenguas extranjeras que no fuera el inglés y la consolidación de programas de formación de profesores con el fin de atender la demanda del sector fuerza trabajadora del tratado. Mercosur representó el parteaguas económico-político-social y cultural para Sudamérica lo que el TLCAN representó para Canadá, Estados Unidos y México.

El portugués se convirtió en una lengua de tendencia en los años noventa en Sudamérica y poco a poco fue tomando espacios hasta convertirse en la lengua de contrapeso con el inglés en países tales como Argentina, Paraguay, Uruguay. En contraparte comenzar una unificación sudamericana a través del uso de dos lenguas (el español y el inglés). En segundo lugar, comenzaron a surgir programas de aprendizaje del portugués en toda la Argentina, Paraguay y Uruguay, en contrapeso el español en Brasil. Después del Mercosur, se visualizaba una movilización de los ciudadanos de esas regiones para unificar el contexto sudamericano y moverse en el mundo lusofonía. En tercer lugar, esta lengua proveniente de las lenguas romances se convertiría en la lengua de la unificación cultural y lingüística en Sudamérica hasta extenderse a México.

Para 1994 la UNAM, ya contaba con un programa de PLE fortalecido con el proyecto de Interacción en el salón de clase de portugués como Lengua Extranjera en el que participaron profesores e investigadores quienes aportaron de manera significativa las mejores estrategias didácticas y buenas prácticas del PLE en México. A la fecha se diseñan programas virtuales de enseñanza del PLE y evaluación (a distancia).

Si bien, en México se comenzó a fomentar el portugués europeo por la fuerte influencia del Instituto Camões ubicado en la Facultad de Filosofía y Letras en la UNAM (2002), la variante del portugués brasileño es la que posee mayor fortaleza y por el número de hablantes es la más enseñada en México. Esto se debe a la relación México-Brasil desde los años sesenta. Ambas variantes dialectales de la lengua portuguesa forman parte del contenido curricular del PLE en los distintos programas que se enseñan en México. En contraparte, en el Centro Cultural Brasileño en México (CCBM) se enseña con la metodología sustentada en los parámetros curriculares del Ministerio de Relaciones Exteriores y la filosofía de enseñanza del "Português nas Unidades da Rede de Ensino do Itamaraty em Países de Língua Oficial Espanhola" (CCBM, 2020). Otros centros e institutos de lenguas extranjeras como Casa do Brasil del Grupo FIGA y DaraIdiomas entre otras organizaciones privadas que también ofrecen cursos al sector empresarial 
que brinda un posicionamiento relevante por la enseñanza de las expresiones culturales lusófonas. Existen más de 14 empresas brasileñas de alto nivel más lucrativas y que tienen un poder económico de Brasil que compite con las empresas mexicanas (Expansión, 2014).

Del año 2000 a la fecha, el portugués ha tenido presencia en universidades públicas y privadas tales como: la Benemérita Universidad Autónoma de Puebla (BUAP), la Universidad Autónoma de la Ciudad de México (UACM), la Universidad Iberoamericana (UIA), la Universidad Veracruzana, la Universidad Autónoma de Nayarit (UAN), la Universidad Juárez Autónoma de Tabasco (UJAT), la Universidad del Claustro de Sor Juana Inés (UCSJ), el Instituto Politécnico Nacional (IPN) y la Universidad Autónoma de Baja California (UABC).

Florêncio da Silva (2018) señala que "los cursos de portugués en México brindan una oferta en el campo de los idiomas extranjeros para responder a las necesidades del contexto internacional. Tienen como objetivo el logro de las competencias generales de comunicación, el desarrollo de la autonomía y el dominio de la interculturalidad de los alumnos". (p. 6).

Dicho lo anterior, las IES mexicanas iniciaron a partir de la firma del TLCAN, los procesos de internacionalización en las universidades mexicanas se agilizaron y se buscaban que los alumnos aprendan una segunda lengua (inglés) y buscar una tercera lengua para aprovechar los intercambios. Brasil representó el destino sudamericano óptimo para formar ingenieros, economistas, profesores, médicos y odontólogos con calidad en las mejores universidades brasileñas. La visión de la Asociación Nacional de Universidades e Instituciones de Educación Superior (ANUIES) a finales de los noventa orientaba a las universidades hacia alternativas de enseñanza para promover el multilingüismo en los programas educativos, el desarrollo de competencias comunicativas efectivas con lenguas adicionales promoviendo la participación de universidades mexicanas universidades brasileñas con la firma del convenio Grupo Coimbra de Universidades Brasileiras (GCUB)-ANUIES en 2014 con el objetivo de entrelazar cooperación académica entres las universidades de Brasil y México.

El Instituto Camões (2021) con sede en Lisboa Portugal señala que en el mundo más de 200 millones de personas hablan portugués y se movilizan en la sociedad lusófona. Sin embargo México ha sido un crisol lingüístico donde el portugués brasileño se ha desarrollado al interior de las universidades desarrollan una política lingüística explícita en los programas de lenguas extranjeras con fines de movilidad estudiantil y académica para atender la política de internacionalización con los países miembros de la lusofonía: Angola, Brasil, Cabo Verde, Mozambique, Guinea Bissau, São Tomé, Príncipe, Portugal, Macau, Timor Oriental, y en partes de la India como Goa, Daman y Diu. En el caso de Baja California sólo nos enfocaremos en los países como Brasil y Portugal dado que en la actualidad la UABC cuenta con más de 20 convenios con universidades lusófonas.

\section{La migración lusófona "At the border region"}

Baja California se ubica en el noroeste de México, colinda al norte con el estado de California en Estados Unidos y es el estado más rico del continente americano. Es la región donde inicia Latinoamérica y espacio geográfico donde se desarrollan expresiones artísticas, culturales, musicales, académicas, emergencia lingüística, económicas y migratorias. Se ha convertido en el paso obligado de la migración para ciudadanos brasileños y haitianos (hablantes del portugués). Las razones por las que se hace atractiva esta zona fronteriza por estar en colindancia con los Estados Unidos y que es una tercera nación en espera de una respuesta afirmativa para recibir visa humanitaria. Aún continúa la construcción del imaginario del "sueño americano" para los migrantes, que consideran que emigrar a los Estados Unidos es fácil y en condiciones óptimas. Sobre los residentes y visitantes portugueses, no se tiene registro en la zona. Sin embargo, hay una vinculación fuerte de las zonas vinícolas con las ciudades productoras vinícolas en "La Ruta del Vino" en el municipio de Ensenada, Baja California. 
Desde la década de los noventa, Tijuana ha sido "ciudad de paso" para los brasileños que quieren migrar a los Estados Unidos. El primer paso es llegar a Tijuana como turistas, para después buscar la oportunidad laboral en la Unión Americana. Entre los empleos que desempeñan son de meseros, cantineros, bus-boys y/o animadores de playa en los hoteles gran turismo, en la zona del Condado de San Diego como La Jolla, Pacific Beach, Coronado y en la ciudad de San Diego en California. La ventaja que tiene la comunidad brasileña es que un alto porcentaje de los migrantes son hablantes del inglés, del español y/o portuñol, además de la lengua materna. En la Era Trump, todo cambió, y las medidas migratorias fueron más estrictas, y el nuevo estatus migratorio para ingresar a Estados Unidos se convirtió para los brasileños más complicado. Esto no ha cambiado con la entrada del gobierno de Joe Bidem.

Las ciudades fronterizas mexicanas de Tijuana, Mexicali, Ciudad Juárez, Laredo, Reynosa son ciudades de población flotante donde llegan migrantes brasileños (por poco espacio de tiempo) para pedir asilo político a los Estados Unidos. Tan sólo en el 2019, según el Instituto Nacional de Migración (INM) en el 2021 un total de 17,900 brasileños han intentado exiliarse en la frontera mexicana y han sido arrestados en los Estados Unidos (El Sol de México, 2019). Para el caso de Tijuana Baja California, se observa una migración masiva de brasileños quienes desean ingresar a Estados Unidos por la vía de obtención de visa humanitaria (en busca de la permanencia migratoria del tercer país en México). De acuerdo con Reyes (2021), de abril a septiembre del año en curso más de 2,000 brasileños han llegado a la frontera para cruzar de manera ilegal y/o solicitar visa humanitaria con base con las estadísticas de deportados de CBP (Aduanas y Protección Fronteriza por sus siglas en inglés), de estos sólo 623 han sido asegurados por el INM. Lo que representa que Baja California tendrá población migrante dado que según los datos de INM se le otorgarán visas humanitarias en México.

En México existe una población de 8,670 brasileños con residencia legal (con base en los datos estadísticos del INM. Los visitantes llegan a México y son registrados como turistas y viaje de negocios. De este rubro hay quienes permanecen en el país y optan por radicar en México y/o migrar a los Estados Unidos y pedir asilo político a Estados Unidos. México se niega a ser tercer país, pero ofrece el programa "Quédate en México" el cual ha sido impulsado por el gobierno de Andrés Manuel López Obrador. Este programa brinda a los extranjeros un permiso de permanencia temporal (visa humanitaria) con oportunidad de obtener un trabajo temporal, lo que les permite esperar en territorio mexicano mientras regularizan su ingreso en acato a las leyes migratorias en Estados Unidos. De no ser aceptados en los Estados Unidos, México será la opción para radicar y comenzar un nuevo estilo de vida.

En el 2019, el INM señaló que "los brasileños no necesitan una visa para su libre tránsito en el país, ya que ellos pueden transitar libremente por territorio nacional y una vez en el norte, intentan de manera ilegal solicitar asilo en el gobierno de Donald J. Trump.” (El Sol de México, 2019). A la fecha, esta medida se encuentra desdibujada de la nueva ley migratoria y sigue siendo rígida con el gobierno del presidente Biden. El turismo recreativo, médico, de negocios, de salud y de la cooperación académica en el ámbito educativo son las agendas relevantes entre la relación México-Brasil.

A partir del 2014, se abren de los vuelos de São Paulo y Río de Janeiro a Ciudad de México y viceversa (Aeroméxico, Avianca, Copa Airlines, LATAM); para privilegiar al turismo brasileño; por ende Tijuana se convierte en la ciudad hub de atractivo para cruzar a los Estados Unidos e ir al "shopping", ya que el turismo brasileño viaja a México con el destino final Tijuana/San Diego; aprovecha la visita a los outlets, disfrutar de los lugares turísticos, sin menoscabar el servicio médico (medicina hospitalaria y estética) que es más económico en esta frontera.

Muñoz Cruz (2010). En su estudio sobre la Educación Intercultural y Política del Lenguaje en nuestro país señala que "México, como ejemplo de país fronterizo con fuerte enclave económico, constituye a un espacio donde confluyen diversas y combinadas dimensiones del fenómeno migratorio transnacional hacia el interior de otra sociedad, cuya economía provoca una 
integración asimétrica, no asumida con el país expulsor de los trabajadores. Actualmente la sociedad huésped-migrante exhibe tres corrientes dominantes: 1) La inmigración de trabajadores hacia el país receptor, 2) El tránsito predominante de otras poblaciones hacia los países desarrollados y 3) El incesante incremento de la inmigración procedentes de una gran diversidad de distintos países". (p.245).

Sin duda, es la cooperación académica entre Brasil y México en la que nos centraremos en este estudio, y la manera como la emergencia de lenguas ha permitido que Baja California fortalezca sus los aspectos socioculturales y lingüísticos a través de los programas de intercambio de la UABC la cooperación académicas con la movilidad académica de profesores e investigadores, intercambio estudiantil semestral hasta por un año, estancias cortas (un mes o más) y aprendizaje del PLE entre otras acciones.

\section{El aprendizaje del portugués en Baja California México}

Toledo (2014) señala que en el 2010 la UABC ofreció por primera vez el programa de Portugués como Lengua Extranjera (PLE) en la Facultad de Idiomas en Ensenada y Tijuana en modalidad de cursos intersemestrales. En el 2012 en el campus Mexicali, se diseña el programa universitario de PLE con el apoyo de la Lic. Berenice Jiménez Hidalgo (UNAM) con el acompañamiento de la Dra. Marisela Colín Rodea (UNAM) y el Dr. Jesús Valdez Ramos (UNAM). A partir de este proyecto institucional, se diseñó el programa de PLE como el documento homologado a nivel estado. Durante ese periodo, se tuvieron cursos de formación docente impartidos por el Dr. Rodrigo Florêncio da Silva como lector de portugués en México adscrito al Instituto Politécnico Nacional (IPN) y la Mtra. Valeria Paola Suárez Galicia por la Universidad Nacional Autónoma de México (UNAM), hoy directora del CCBM. Sin duda, la UNAM y el IPN fueron las instituciones que a través de convenios de colaboración brindaron los primeros cursos de formación de profesores de PLE en la UABC.

El programa de PLE se implementó en la UABC en el periodo rectoral del Dr. Gabriel Estrella Valenzuela (2006-2010) con el objetivo de integrar a la universidad al consorcio de universidad extranjeras y por ende ofrecer una lengua extranjera adicional a los 6 programas (alemán, chino mandarín, francés, inglés, italiano y japonés) que se impartían en el Centro de Idiomas dependiente de la Facultad de Idiomas de la UABC. El portugués representó el nuevo lenguaje (new language) que habilitaría al universitario con la competencia lingüística lusófona para participar en el intercambio estudiantil a países como Brasil y Portugal. Con esto, se cubriría la demanda de estudiantes universitarios que aspiran a los programas de becas que ofrecen las universidades de los consorcios educativos Erasmus Mundus, Becas del gobierno brasileño y Becas Santander (LATAM).

En el 2014, en el periodo rectoral del Dr. Felipe Cuamea Velázquez UABC se modificó la estructura del Centro de Idiomas adscrito a la UABC (en todo el estado en las 4 sedes) y se convierte a una entidad asociada denominada UNISER UABC A.C., como marca registrada adquiere el nombre de UNISER, A.C. (División Universitaria de Lenguas Extranjeras). En este periodo, se fortaleció el PLE con la llegada de los profesores de dicha lengua que se habían formado en Portugal y Brasil. Es así como el portugués ocupa la quinta posición con mayor opción para la acreditación de una lengua extranjera como requisito de egreso con base en artículo 116 del Estatuto Escolar de la UABC (2018, p.13).

Según los datos de alumnos inscritos en UNISER, A.C. (2021), del 2010 al 2021 han estudiado en dicho centro 847 alumnos del nivel en los diferentes niveles que se enseñan del básico al intermedio (A1 al B1 del Marco Común Europeo de Referencia para las Lenguas). Esto es una muestra del interés que existe por parte de los alumnos de participar en las convocatorias de intercambio estudiantil que ofrece la UABC desde que existe el PLE en la para la formación de los primeros cuadros de profesores de PLE. Además de continuar estudiando la lengua de manera virtual a causa de la contingencia sanitaria de la Covid-19. 
Otro hecho representativo en el crisol lingüístico de la migración en Baja California ha sido la comunidad de haitianos educados que radican en la frontera. Esta nueva migración ha traído una nueva amalgama lingüística de hablantes del portugués y del criollo (francés) de Haití en Baja California. Para Llenderosas (2006), su postura sobre la reconstrucción de Haití se basa en la participación de la misión de paz MINUSTAH, propuesta por la Organización de las Naciones Unidas (ONU) el 01 de junio de 2004. Brasil aportó una gran fuerza política y de conocimiento para formar cuadros que revitalizaron Haití y por ende el componente lingüístico estaba incluido en la reconstrucción de la isla devastada.

Dicho lo anterior, la población haitiana tomó del comando militar brasileño las mejores prácticas en medicina, docencia, ingeniería civil, enfermería y política pública para reconstruir Haití después de los sismos y huracanes. Lo más esencial fue que la comunidad haitiana aprendió el portugués como tercera lengua, adicional al criollo que hablan como lengua de socialización y el francés (variante dialectal haitiano) que algunos habitantes radicados en Baja California hacen de estas lenguas emergencias lingüísticas de la franja fronteriza.

"Hay una realidad que afronta esta lengua en la región. A partir de la migración haitiana a la localidad, y de acuerdo con el proyecto Archivo con testimonios de los Migrantes, desarrollado por el Colegio de la Frontera Norte en el año 2016. En la actualidad existe un grupo de 1057 haitianos registrados, de los cuales 200 de ellos hablan portugués, debido a que participaron en misiones de jornadas laborales en Brasil. (Toledo Sarracino y García-Landa, p. 90)”

Dos Santos Silva (2021) señaló que la escuela privada que ha construido desde el 2018 en Tijuana de aprendizaje de portugués denominada Thales do Brasil tiene como objetivo conformar un colectivo cultural-educativo en la frontera México-Estados Unidos. Los grupos presenciales son pequeños y ha alcanzado un total 170 egresados del PLE (desde básico hasta avanzado). Extendiéndose los programas híbridos (presencial y virtual) como consecuencia de la pandemia de la Covid-19 que amenaza a nivel mundial. El PLE que promueve la escuela tiene un alcance a 19 países con un total de 260 estudiantes inscritos incluyendo a los estudiantes de Baja California. Esto representa el crecimiento de los interesados por aprender el portugués con fines turísticos, académicos y laborales.

\section{Los programas educativos de portugués lengua extranjera}

Como parte de los procesos de internacionalización de la UABC, en octubre de 2019 bajo el periodo rectoral del Dr. Daniel Octavio Valdez Delgadillo se firmó el convenio de colaboración entre la UABC y la Asociación Nacional de Instituciones de Educación Superior (ANUIES) para afiliar a la Universidad al Programa de Intercambio Académico Brasil-México (BraMex) de movilidad estudiantil que surgió en México en el 2014. Este consorcio tuvo mayor cobertura hasta el 2019 donde incrementó el número de universidades mexicanas se incorporaron con el apoyo de la ANUIES. Además, se firmó en el 2021, el primer convenio que tiene la UABC de cooperación académica con la Universidad de Cuanza en Angola en África.

Al 2021, existen más de 300 estudiantes y 40 profesores que han participado en movilidad internacional en países lusófonos. Las instituciones participantes en estas iniciativas fueron: Universidade Estadual de Campinas (UNICAMP), Universidade de São Paulo (USP), Universidade Católica de São Paulo (UCSP), Instituto Federal de Educación, Ciencia y Tecnología (IFSP) en Sul de Minas Gerais, Instituto Euro-Americano de Educación y Motricidad Humana, Universidade de Ouro Preto (UOP), el Instituto Federal (IF) con sedes en Rio de Janeiro, São Paulo, Hortolândia y Campinas, Universidade Federal de Pernambuco (UFP), Universidade Estadual de Pernambuco (UEP), etc. Además que para la Unión Europea, Portugal representa el vínculo lusófono con el Instituto de 
Artes Visuales, Diseño y Mercadotecnia de Portugal, el Instituto Politécnico de Bragança (IPB), Universidade de Lisboa (UL) entre otras universidades.

\section{Metodología}

Este estudio de tipo descriptivo-analítico se analizan las narrativas de 4 profesores quienes participaron en los cimientos de la enseñanza del PLE en la región fronteriza desde un enfoque glotopolítico de Guespin y Marcellesi (1986) y Lagares (2018) donde se profundiza al lenguaje desde un elemento esencial de la cultura y la interacción entre sus hablantes. Además, frente a los nuevos escenarios de política de lengua y las nuevas formas de comunicación en un mundo global, se refleja la naturaleza de la lengua y la naturaleza de las narrativas (Estrela, 2018). Por ello, a partir de este análisis de las narrativas de los informantes, se identificarán 3 elementos tales como: 1) Lenguaje como elemento sociocultural, 2) Interacción entre los hablantes y 3) Efectos glotopolíticos en el PLE.

Para este estudio se tiene como sujetos informantes que fueron profesores pioneros en la formación de los primeros cuadros de profesores expertos en PLE en Baja California, México. Esta formación inició desde el 2014 cuando egresaron las primeras generaciones del programa de lengua portugués un total de 787 alumnos distribuidos en niveles básico, intermedio e intermedio alto. Lo que ha representado a la fecha que más de 158 alumnos participen en programas de intercambio estudiantil con universidades socias con las que institución tiene convenios de colaboración en Brasil y Portugal, lo que ha significado un impacto significativo en el posicionamiento de la dicha lengua en la última década dado a los indicadores de internacionalización de la UABC.

Tabla 1. Perfil de sujetos de los entrevistados y nivel de participación en el programa de PLE.

\begin{tabular}{|l|l|l|c|l|}
\hline & Sexo & \multicolumn{1}{|c|}{ Perfil } & $\begin{array}{c}\text { Años de } \\
\text { experiencia en } \\
\text { la enseñanza } \\
\text { del PLE }\end{array}$ & Nivel de participación del PLE en la UABC \\
\hline S1 & F & Profesora de PLE/Lic. en Letras (UNAM) & 10 & $\begin{array}{l}\text { Participó como profesora y diseñadora del curso } \\
\text { de portugués en la UABC en 2011. }\end{array}$ \\
\hline S2 & F & $\begin{array}{l}\text { Doctora en Lingǘrtica (UPF), profesora } \\
\text { investigadora y formadora de profesores } \\
\text { de lenguas extranjeras (UNAM) }\end{array}$ & 40 & $\begin{array}{l}\text { Participó en el programa de formación de maestros } \\
\text { en lenguas modernas como profesora invitada en } \\
\text { 2010. }\end{array}$ \\
\hline S3 & M & $\begin{array}{l}\text { Doctor en Ciencias (IPN), SNI I, Profesor } \\
\text { investigador y formador de profesores de } \\
\text { lenguas extranjeras (IPN) }\end{array}$ & 12 & $\begin{array}{l}\text { Participó en el programa de formación de } \\
\text { profesores de PLE como profesor invitado en } \\
\text { 2010. }\end{array}$ \\
\hline S4 & M & $\begin{array}{l}\text { Profesor de PLE/Maestro en Estudios } \\
\text { Socioculturales (COLEF) }\end{array}$ & $\begin{array}{l}\text { Forma parte de la planta de docentes del programa } \\
\text { PLE en UNISER, UABC A.C desde el 2018. }\end{array}$ \\
\hline
\end{tabular}

Fuente: Elaboración propia.

\section{Resultados y Análisis}

Para realizar este estudio se realizaron análisis de las narrativas bajo el modelo glotopolítico de Guespin y Marcellesi (1986, p. 5) y Lagares (2018) que señalan que es necesario englobar todos los hechos del lenguaje en cada acción de la sociedad para revestirlo en lo político. Por ende, a partir del análisis de las narrativas de los informantes, se identificarán los señalamientos de cada uno de los actores realizaron para dar cumplimiento a los tres criterios establecidos en la Taxonomía (Ver Tabla 2) determinada en este estudio tales como lengua como un agente de comunicación comunitaria, con funcionalidad internacional o con funcionalidad educativa tal como lo señala Lagares (2018, pp. 63-65). 
A los sujetos participantes se le hicieron preguntas relacionadas a su participación en la operación del PLE en la UABC a) ¿Cuándo inició el proyecto de portugués en la UABC? b) el número de alumnos participantes a la fecha desde que iniciaste y su impacto c) ¿Qué opina sobre el futuro del portugués en Baja California?

A continuación, se presentan los componentes glotopolíticos identificados en las narrativas donde de manera explícita los participantes cumplen con los tres componentes tales como 1) Lenguaje como elemento esencial de la cultura, 2) Interacción entre los hablantes y 3) Efectos glotopolíticos.

Tabla 2. Taxonomía glotopolítica Lagares (2018) para el programa de PLE en la UABC.

\begin{tabular}{|c|c|c|c|}
\hline & $\begin{array}{l}\text { Lenguaje como elemento esencial } \\
\text { de la cultura }\end{array}$ & Interacción entre los hablantes & Efectos glotopolíticos \\
\hline S1 & $\begin{array}{l}\text { Los estudiantes siempre fueron } \\
\text { muy cooperativos para trabajar } \\
\text { aspectos culturales y de la lengua, } \\
\text { para apoyar en las actividades de } \\
\text { difusión, para apoyar el trabajo del } \\
\text { centro de idiomas y de este caso } \\
\text { específico de las clases de portugués. }\end{array}$ & $\begin{array}{l}\text { Tuve la oportunidad de interactuar } \\
\text { con los estudiantes y me brindaron } \\
\text { también al estar ahí dos horas, } \\
\text { todos los días, durante tres años. } \\
\text { Tuvimos una buena apertura } \\
\text { para que aprendieran las dos } \\
\text { variantes, la brasileña y la } \\
\text { portuguesa en el mismo } \\
\text { programa que se construyó. }\end{array}$ & $\begin{array}{l}\text { Una nueva manera de observar a la lengua } \\
\text { portuguesa en una zona geográfica donde sólo } \\
\text { se ubica al inglés como segunda lengua no fue } \\
\text { nada fácil. Por ende, se me abrieron las puertas } \\
\text { con otras escuelas y empresas para impartir } \\
\text { cursos de portugués. Las empresas buscaban y } \\
\text { creo que al final esta visión que usted tuvo de } \\
\text { abrir el portugués en la Facultad de Idiomas } \\
\text { Mexicali, posteriormente también abrirlo en } \\
\text { Tijuana en Ensenada. Este empuje que } \\
\text { dieron a la economía en Baja California, } \\
\text { que lo colocó nuevamente, en el mapa, no } \\
\text { nada más por unas cuestiones relacionadas } \\
\text { con seguridad, sino con realmente ser un } \\
\text { foco de atracción económica y de un modo } \\
\text { de vida diferente. }\end{array}$ \\
\hline $\mathrm{S} 2$ & $\begin{array}{l}\text { La historia del portugués en la } \\
\text { ENALLT, antes el CELE de la } \\
\text { UNAM, sí, evidentemente fue un } \\
\text { inicio con profesores exiliados DEL } \\
\text { BRASIL y con personas que habían } \\
\text { hecho recientemente una maestría en } \\
\text { el área de psicolingüística. Esto le } \\
\text { diÓ un perfil muy especial al } \\
\text { profesorado, pues se estaba como en } \\
\text { el espacio, un tanto crítico, por la } \\
\text { situación social de Brasil en los } 60 \text { 's } \\
\text { y al mismo tiempo en los enfoques } \\
\text { más cognitivos, que era en ese } \\
\text { momento el gran teórico y que va a } \\
\text { permitir inicialmente pues } \\
\text { acompañar la metodología que } \\
\text { estaba de moda en esa época que } \\
\text { eran los cursos audiovisuales y de } \\
\text { cultura. }\end{array}$ & $\begin{array}{l}\text { Brasil tiene muchísimos } \\
\text { hablantes, no está preocupado por } \\
\text { lo que le pase al portugués, pero } \\
\text { cuando con el Mercosur en el año } \\
\text { 1990, ahí es cuando el Mercosur } \\
\text { pone en la mesa el papel de la } \\
\text { lengua, como ha pasado en otros } \\
\text { espacios, en otros tratados, y } \\
\text { entonces viene como un tipo de } \\
\text { reciprocidad entre los gobiernos } \\
\text { de Uruguay y de Argentina, de } \\
\text { enseñar portugués a nivel básico } \\
\text { medio y de enseñar español, en el } \\
\text { caso de Brasil, digamos que la } \\
\text { actitud generalmente de los } \\
\text { brasileños y de los propios } \\
\text { hispanohablantes no era aprender } \\
\text { ni portugués ni español. Las } \\
\text { creencias siempre eran para } \\
\text { que, si nos entendemos, entonces } \\
\text { este sí nos entendemos era falso, } \\
\text { nada fácil, una falsa impresión, } \\
\text { porque al final la comunicación } \\
\text { tenía muchos malentendidos y se } \\
\text { quedaba en un portuñol o en } \\
\text { algún en alguna otra forma al } \\
\text { revés, del portugués hacia el } \\
\text { español. Entonces esto, pues } \\
\text { digamos, ha habido ese mercado, } \\
\text { Mercosur no tuvo un interés por } \\
\text { México, definitivamente, pero } \\
\text { siempre existió un diálogo con, } \\
\text { pues con los centros de } \\
\text { investigación de Brasil y con las } \\
\text { universidades. }\end{array}$ & $\begin{array}{l}\text { Se instalan en México muchas empresas. Y } \\
\text { hay mucha migración, tras migración de } \\
\text { México hacia Estados Unidos, de brasileños. } \\
\text { Entonces digamos que esto es un elemento que } \\
\text { va a hacer que el portugués adquiera otro } \\
\text { carácter, no sólo para estudiar, sino que sea } \\
\text { una lengua para trabajar, en una lengua de } \\
\text { trabajo. Ahí es donde el Instituto Camôes } \\
\text { apoya con la Facultad de Filosofía, la creación } \\
\text { de la licenciatura en Letras Portuguesas y se } \\
\text { llama así Licenciatura en Letras Portuguesas. } \\
\text { Y bueno, aunque hay profesores brasileños, } \\
\text { tiene una formación de la variante portuguesa. }\end{array}$ \\
\hline
\end{tabular}




\begin{tabular}{|c|c|c|c|}
\hline S3 & $\begin{array}{l}\text { Mi alianza con la UABC empezó en } \\
2011 \text {, es primer acercamiento, yo } \\
\text { todavía era lector de Brasil en } \\
\text { México y fueron cursos que se } \\
\text { dieron a los estudiantes de portugués } \\
\text { en la UABC. En ese entonces estaba } \\
\text { la profesora Berenice Jiménez, } \\
\text { empezaba con el programa, después } \\
\text { se dieron cursos y esa capacitación, } \\
\text { si no me equivoco, era la profesora } \\
\text { que estaba a cargo y con los alumnos } \\
\text { en diferentes carreras Hablaba } \\
\text { acerca de la cultura brasileña, de } \\
\text { la cultura, eso en } 2011 \text {. Ya en } 2012 \\
\text { hubo ya congresos donde yo fui } \\
\text { también para participar, y habla } \\
\text { entonces de la enseñanza de } \\
\text { portugués en México, ya también } \\
\text { como forma de actuación, de } \\
\text { actividades para la difusión de la } \\
\text { lengua portuguesa. Ya en } 2012 \\
\text { 2013, yo fui ya a capacitar } \\
\text { profesores a la UABC, había } \\
\text { alumnos que habían sido profesores } \\
\text { empíricos y que habían sido alumnos } \\
\text { de portugués y fueron siete } \\
\text { profesores inicialmente en Baja } \\
\text { California y después participaron en } \\
\text { un curso de } 40 \text { horas durante una } \\
\text { semana. Después continuaron los } \\
\text { cursos de formación de lengua y } \\
\text { cultura en visitas posteriores. }\end{array}$ & $\begin{array}{l}\text { En la UABC se dieron distintos } \\
\text { programas de formación de } \\
\text { profesores de portugués para tener } \\
\text { los primeros cuadros de docentes } \\
\text { que enseñan la lengua. Recuerdo } \\
\text { que, fueron diferentes grupos } \\
\text { para hablar acerca de Brasil, } \\
\text { tanto de maestría y también de } \\
\text { otras carreras la cual para dar } \\
\text { una plática acerca de Brasil. La } \\
\text { interacción social era lo más } \\
\text { importante en la lengua } \\
\text { portuguesa. Me acuerdo también } \\
\text { que hubo la participación del Dr. } \\
\text { Marco Bagno quien también } \\
\text { participó de sesiones de formación } \\
\text { de futuros maestros y además } \\
\text { sirvió para hablar claro de la parte } \\
\text { lingüística y su teoría. Sin } \\
\text { embargo, también recuerdo el } \\
\text { trabajo del Dr. Gabriel Leopoldino } \\
\text { dos Santos quien vino a dar una } \\
\text { mirada de la política lingüística y } \\
\text { la enunciación. Esto significó } \\
\text { mucho para un nuevo grupo de } \\
\text { profesores que se formaban bajo } \\
\text { una nueva escuela lingüística y } \\
\text { que creció el programa. }\end{array}$ & $\begin{array}{l}\text { El portugués es un idioma que está } \\
\text { creciendo mucho por la búsqueda de la } \\
\text { unificación lusófona, precisamente en } 2014 \\
\text { y 2016, fue donde creció por todos los } \\
\text { avances que tenía el Brasil en ese entonces, } \\
\text { tanto la parte económica y por la presidenta } \\
\text { Dilma Rousseff, que también incentivó } \\
\text { mucho que empresas vieran a Brasil con } \\
\text { otros ojos. También los de eventos } \\
\text { deportivos del 2014 y 2016 dieron } \\
\text { reflectores a Brasil. Los eventos de deportes } \\
\text { como las Olimpiadas en } 2016 \text { y en 2014 del } \\
\text { Mundial de Fútbol donde Brasil fue centro } \\
\text { de mundo; y mucha gente querían aprender } \\
\text { portugués por el turismo y también por } \\
\text { temáticas de trabajo, y yo veo en ese caso } \\
\text { también la importancia de hacer un estudio } \\
\text { en Brasil. Un Intercambio estudiantil creo que } \\
\text { también fue algo que veo qué se puede en } \\
\text { Baja California crece muchísimo. Hay interés } \\
\text { de Brasil, no solamente portugués brasileño } \\
\text { sino como el portugues que se habla en } \\
\text { Portugal, como también los países que está en } \\
\text { África, como Angola, como Mozambique, } \\
\text { como Cabo Verde, donde la gente puede tener } \\
\text { oportunidad también. Entonces, no veo } \\
\text { únicamente portugués para Brasil, para } \\
\text { Portugal, veo portugués global con } \\
\text { diferentes oportunidades en el mundo muy } \\
\text { general que puede desarrollar, nos lleva eso } \\
\text { técnica para trabajar en países africanos. } \\
\text { Entonces, como veo, el futuro del portugués } \\
\text { Baja California es prominente y espero que } \\
\text { existan mejores oportunidades para los } \\
\text { estudiantes y egresados de la UABC. }\end{array}$ \\
\hline $\mathrm{S} 4$ & $\begin{array}{l}\text { Yo creo que, si no hay una persona } \\
\text { para fomentar el portugués } \\
\text { realmente en Baja California, una } \\
\text { persona con un perfil cultural de } \\
\text { Brasil es muy difícil que el proyecto } \\
\text { siga ya que enseñar portugués es un } \\
\text { mercado virgen en Baja California. } \\
\text { Como una persona que sabe la } \\
\text { lengua y la cultura, fomento la } \\
\text { cultura brasileña en Tijuana con } \\
\text { clases de portugués, yo veo mucho } \\
\text { futuro para este idioma en Baja } \\
\text { California ya que es un estado que } \\
\text { tiene una diversidad cultural. Yo no } \\
\text { veo el portugués como una primera } \\
\text { opción ni como segunda opción. Yo } \\
\text { veo como tercera, cuarta, quinta } \\
\text { opción que después de otros } \\
\text { idiomas, entonces, pero también es } \\
\text { interesante ver y si lo puedo revisar, } \\
\text { que sí han salido muchas } \\
\text { oportunidades de trabajo. }\end{array}$ & $\begin{array}{l}\text { Yo creo que el portugués genera } \\
\text { una sensación de comunidad en } \\
\text { Tijuana educada y por eso la } \\
\text { gente sigue estudiando, porque } \\
\text { muchos alumnos que, aunque ya } \\
\text { lo tengan, que lo terminen, pues } \\
\text { ellos pues quieren seguir } \\
\text { practicando, desarrollando su } \\
\text { idioma. }\end{array}$ & $\begin{array}{l}\text { Mucha me manda mensajes de textos o correos } \\
\text { porque quieren que los contacte con hablantes } \\
\text { de la lengua. Una empresa brasileña } \\
\text { "Pancroni" así se llama, creo requiere gente } \\
\text { que hable inglés, español y portugués.He } \\
\text { buscado personas entre mis ex- alumnos que } \\
\text { hablen portugués u otras lenguas, es más las } \\
\text { empresas que me buscan son call centers y } \\
\text { maquiladoras. Se necesita gente políglota Se } \\
\text { ha diversificado la manera de emplear en } \\
\text { las empresas en Baja California. Entonces el } \\
\text { interés se da y yo creo que ya hablé mucho. } \\
\text { Esto ha transformado la cultura de } \\
\text { contratación. }\end{array}$ \\
\hline
\end{tabular}

Fuente: Elaboración propia a partir de las entrevistas a Colin (2021), dos Santos (2021), Florêncio da Silva (2021) y Jiménez (2021).

\section{Discusión}

En primer momento analizaremos el papel que juega el portugués como elemento esencial de la cultura bajacaliforniana y el proceso formativo del universitario. Por un lado, la universidad se preocupa por mantenerse en los rankings internacionales y el boom de la internacionalización de las universidades en el siglo XXI, en consecuencia, es necesario que sus estudiantes y académicos 
participen en convocatorias internacionales y de divulgación científica. Por tal motivo, se presentan las narrativas de profesores expertos en PLE quienes fueron parte del parteaguas de la enseñanza del portugués en la región fronteriza:

S1 "La Universidad Autónoma Baja California, respondía a esta necesidad de los alumnos que estaban entrando en este proceso de internacionalización. Ya había habido alumnos y profesores que habían viajado a Brasil y uno que otro a Portugal, de hecho, habían establecido contacto cultural, digamos, sin saber hablar la lengua, y bueno, ya tenían una experiencia".

S2: "Tuve el honor y la oportunidad de abrir estos cursos de portugués en la UABC hace exactamente diez años, cuando se dió este acercamiento y posteriormente de una entrevista con la dirección de la Facultad, sostuvimos la invitación a formar parte del equipo de lo que era el Centro de Idiomas de la Facultad de Idiomas Campus Mexicali de la Universidad Autónoma de Baja California".

Sin duda, la participación de los expertos en PLE en el 2010 en la región fronteriza cimentó las bases de la enseñanza de la lengua portuguesa en el noroeste de México. Esta acción de la política lingüística universitaria cimentó las bases y abonó al abanico lingüístico en Baja California.

S4 "Creo que sí es importante promover la lengua portuguesa en la región, no hay una persona más apropiada que los que nos dedicamos a enseñar y fomentar el portugués en Baja California. quien se dedique a ello debe ser una persona con un perfil cultural y conocimiento de Brasil y los países donde se habla la lengua. Es muy difícil que un proyecto cultural de la lengua siga si no hay binomio cultura-lengua. Por ello, insisto, enseñar portugués es abrir lazos con Brasil y el mundo, crear un espacio académico poco explorado en Baja California que debe fortalecerse.

Por ello, es pertinente promover una política lingüística explícita e implícita en las instituciones educativas mexicanas que tengan programas coherente y congruente acorde con las necesidades del entorno social, político, económico y empresarial a fin de desarrollar los programas de estudios y preparar a los estudiantes para la participación en el intercambio estudiantil y aprendizaje a lo largo de toda la vida.

\section{Conclusiones}

El portugués como lengua de acceso en Baja California continúa extendiéndose a lo largo de todo el estado en los cinco municipios del estado fronterizo con los Estados Unidos, y su alcance en los campos académicos turísticos es significativo para la región en una integración glotopolítica. Sin embargo, bastaría observar en los próximos años ¿Cuál será el impacto real de todos los lusohablantes? ¿Cómo la relación México-Brasil se fortalecerá a través del entendimiento mutuo de la lengua y la cultura? Pero lo más relevante es que en esta región fronteriza ya comienza a crecer el número de hablantes que han optado por el portugués como su segunda o tercera lengua. La UABC tiene una responsabilidad compartida con la academia fronteriza y con las instancias que promueven la enseñanza de la lengua portuguesa. En los Estados Unidos, para el caso específico en el estado de California en Estados

Unidos, la San Diego State University cuenta con el Departamento de Español y Portugués además que comparte la misma ideología con la University of California San Diego, ambas al igual que la UABC tienen la misión de enseñar lenguas extranjeras a través de los programas de portugués para universitarios en los centros de lenguas donde se enseña también PLE a la comunidad norteamericana. Dicho lo anterior, entre Baja California y California se comparten intereses en común en la construcción de 
comunidades de habla multiculturales y multilingües; además existen instituciones de prestigio en la región CaliBaja (región conformada por estos dos estados fronterizos dirigida al sector industrial y de desarrollo económico binacional).

Por ello se hacen las siguientes consideraciones finales:

1. Fortalecer la formación integral de los estudiantes universitarios con el aprendizaje de más de una lengua extranjera para fines de integración y movilidad social internacional.

2. Promover la multiculturalidad en las comunidades académicas de la frontera México-Estados Unidos.

3. Estrechar lazos de colaboración entre las organizaciones que promueven el aprendizaje de la lengua portuguesa en Ciudad de México y la región Calibaja (California y Baja California).

4. Fortalecer los programas de PLE y capacitación docente.

5. Afianzar los convenios de colaboración con las universidades brasileñas y portuguesas, además con otras universidades integrantes del mundo lusófono.

6. Conformar alianza estratégica entre las universidades fronterizas que enseñan PLE para construir una comunidad de enseñanza de la lengua portuguesa en la frontera México-Estados Unidos.

En concordancia con las acciones antes mencionadas, no podrán realizarse sin el esfuerzo deliberado de los gestores y/o planificadores lingüísticos desde las universidades y las generaciones que se formen en el área de PLE, ya que que como observadores de una sociedad multicultural y multilingüe aportan y articulan acciones concretas para conformar un contexto glotopolítico del PLE en esta región fronteriza (México-Estados Unidos) denominada CalBaja.

Finalmente, este estudio se considera el primero en enmarcar la parte históricas y los requerimientos académicos vistos desde la glotopolítica en la Universidad Autónoma de Baja California que permitirá desarrollar en el grupo de investigación denominado Cuerpo Académico Política Lingüística y Enseñanza de Lenguas reconocido por el Programa de Desarrollo Profesional (Prodep) de la Secretaría de Educación Pública (SEP) donde se formularán nuevas líneas de investigación y realización de trabajos a futuro para documentar la historia de la enseñanza de lenguas en México, el seguimiento que se tenga de la evolución de los programas desarrollados y su aplicación en el contexto de toma de decisiones en las universidades del sector público y privado.

\section{Referencias}

Arnoux, E. N. de (2008). Ámbitos para el español: recorridos desde una perspectiva glotopolítica. Revista de Estudos e Reflexões Tecnológicas da Faculdade de Indaiatuba, 6, 1-28. Recuperado de https://reverte.fatecid.com.br/index.php/revista/article/view/25/29

Bufi Zanon, S. (2020). Fundación del Centro de Enseñanza de Lenguas Extranjeras de la Universidad Nacional Autónoma de México. Universidades, 65(61), 3752. https://doi.org/10.36888/udual.universidades.2014.61.314

Centro Cultural Brasil-México (2020). Programa de Enseñanza de Portugués Lengua Extranjera. CCBM:México.

Colin-Rodea, M. (2021). El portugués en la Universidad Nacional Autónoma de México. /Entrevistada por David Guadalupe Toledo Sarracino. Universidad Autónoma de Baja California.

Estrela, C. (2018). Metodología Científica:Ciencia, Ensino, Pesquisa. Editores Artes Médicas.

Muñoz Cruz, H. (2010). Educación Intercultural y Política del Lenguaje en México. En Terborg y García Landa (2010) (Coords.). Los restos de la planificación del lenguaje en el siglo XXI. UNAM:México.

De Beer, G. (1974). Language policy in Mexico: De jure and de facto. Bilingual Review / La Revista Bilingüe, 1(2), 203-207. Retrieved March 30, 2021, from http://www.jstor.org/stable/25743589

Dalpiaz, R. \& Stanzani, L. (2012). Una experiencia contada: La enseñanza del portugués como lengua extranjera a colombianos en Bogotá. ESPIRAL. Revista de Docencia e Investigación. 2 (1), 21-26.

Dos Santos Silva, T.W. (2021). El aprendizaje del portugués en Tijuana/Entrevistado por David Guadalupe Toledo Sarracino. Universidad Autónoma de Baja California. 
Florêncio da Silva, R. (2018). O uso das TIC e da interculturalidade no ensino do idioma português no México. Nonada, Porto Alegre, 1(30), pp. 25-39.

Almeida Filho, J.C. (1992). Relatório de Pesquisa:projeto de interação e relevância no ensino de línguas em Campinas. UNICAMP:Brasil.

González, R. (31 de enero de 2020). México da chance a los brasileños. El Sol de México. https://www.elsoldemexico.com.mx/mexico/sociedad/mexico-da-chancea-brasilenos-dice-sre-4775251.html

Guespin, J. \& Marcellesi, J.B. (1986). Pour la Glottopolitique. Languages. 1 (83), 21-31.

Instituto Camões (2021). Aprendizaje del portugués en el mundo. Instituto Camões:Lisboa.

Jiménez, B. (2021). El portugués en la Facultad de Idiomas de la UABC. Entrevistado por David Guadalupe Toledo Sarracino. Universidad Autónoma de Baja California.

Lagares, X. (2018). Qual política lingüística? Desafios glotopolíticos contemporáneos. Parabola:Brasil.

Llenderosas, E. (15-18 de marzo de 2006). Argentina, Brasil y Chile en la reconstrucción de Haití: Intereses y motivaciones de la participación en conjunta. Meeting of the Latin American Studies Association. San Juan, Puerto Rico.

Herrera, M. E. (2006). La Enseñanza de lenguas extranjeras en el CELE de la UNAM. Reencuentro. Análisis de Problemas Universitarios 1 (47), pp 9-16 [Fecha de Consulta 30 de marzo de 2021]. Disponible en http://www.redalyc.org/articulo.oa?id=34004702

Reyes, K. (13 de noviembre de 2021). Aumenta en BC la detención de brasileños cruzando a Estados Unidos. El Imparcial.

dhttps://www.elimparcial.com/tijuana/tijuana/Aumenta-en-BC-detencion-de-brasilenos-cruzando-a-EU-20211112-0031.html

Toledo Sarracino, D. G. (29-30 de octubre de 2014). La política lingüística del Portugués Lengua Extranjera en la UABC. (Ponencia). Congreso CIPLE, Ciudad de México, México.

Toledo Sarracino, D. G. \& García Landa, L. (2018). Escenarios lingüísticos emergentes en la frontera tijuana-san diego. Káñina, 42(2), 87111. https://dx.doi.org/10.15517/rk.v42i2.34597

UNISER, A.C. (2021). Reporte impreso de matrícula del Portugués Lengua Extranjera. UABC:México.

UABC (2028). Estatuto Escolar de la Universidad Autónoma de Baja California. UABC:México.

UNAM (2021). Reseña histórica de la enseñanza del portugués en la UNAM. https://enallt.unam.mx/index.php?categoria=2\&subcategoria=13\&contenido=279 